\title{
PROBLEMS AND RESULTS ON ADDITIVE PROPERTIES OF GENERAL SEQUENCES. I
}

\author{
P. ERDŐS AND A. SÁRKÖZY \\ Dedicated to the Memory of E. G. Straus
}

Let $a_{1}<a_{2}<\cdots$ be an infinite sequence of positive integers and denote by $R(n)$ the number of solutions of $n=a_{l}+a_{j}$. The authors prove that if $F(n)$ is a monotonic increasing arithmetic function with $F(n) \rightarrow+\infty$ and $F(n)=o\left(n(\log n)^{-2}\right)$ then $|R(n)-F(n)|=$ $o\left((F(n))^{1 / 2}\right)$ cannot hold.

1. Let $\mathscr{A}=\left\{a_{1}, a_{2}, \ldots\right\}\left(a_{1}<a_{2}<\cdots\right)$ be an infinite sequence of positive integers. Denote by $R(n)$ the number of solutions of $n=a_{t}+a_{J}$. Sidon asked more than 50 years ago if there is a sequence $\mathscr{A}$ for which for every $n>n_{0}$,

$$
R(n)>0 \text { but for every } \varepsilon>0, \quad R(n) / n^{\varepsilon} \rightarrow 0 ?
$$

By use of probabilistic methods P. Erdős proved the following much stronger result:

There is a sequence $\mathscr{A}$ so that there are two constants $c_{1}$ and $c_{2}$ for which for every $n$

$$
c_{1} \log n<R(n)<c_{2} \log n .
$$

It is still a challenging problem to give a constructive proof of (2) or even of (1). We can make no contribution to this problem at the moment. An old conjecture of Erdős states that for no sequence $\mathscr{A}$ can we have

$$
R(n) / \log n \rightarrow c \quad(0<c<+\infty) .
$$

We cannot attack (3) at the moment but we can prove that if $G(n)$ is monotonic, $G(n) \rightarrow+\infty$ and $G(n)$ is of regular growth then

$$
R(n) / G(n) \log n \rightarrow c \quad(0<c<+\infty)
$$

is possible. In view of the difficulty of (3) two questions are natural. Can one prove that for every $\mathscr{A}$

$$
\limsup _{n \rightarrow+\infty}|R(n)-\log n|=+\infty,
$$

and is it true that for every $(0<) c_{1}<1<c_{2}$

$$
c_{1} \log n<R(n)<c_{2} \log n
$$


is possible? (6) which is weaker than (3) but stronger than (2) remains open, but we can prove (5) and in fact we can show that

$$
|R(n)-\log n| /(\log n)^{1 / 2} \rightarrow 0
$$

is also impossible.

More generally, we will prove the following theorem:

THEOREM. If $F(n)$ is an arithmetic function such that

$$
\begin{gathered}
F(n) \rightarrow+\infty, \\
F(n+1) \geq F(n) \quad \text { for } n \geq n_{0}, \\
F(n)=o\left(n(\log n)^{-2}\right),
\end{gathered}
$$

and we write

$$
\Delta(N)=\sum_{n=1}^{N}(R(n)-F(n))^{2}
$$

then

$$
\Delta(N)=o(N F(N))
$$

cannot hold.

The following corollary is a trivial consequence of the theorem above:

COROLlary. If $F(n)$ is an arithmetic function satisfying (8), (9) and (10), then

$$
\max _{n \leq N}|R(n)-F(n)|=o\left((F(N))^{1 / 2}\right)
$$

cannot hold.

(So that e.g.

$$
R(N)-B(\log N)^{C}=o\left((\log N)^{C / 2}\right), \quad B>0, C>0
$$

or

$$
R(N)-B N^{C}=o\left(N^{C / 2}\right), \quad B>0,0<C<1
$$

cannot hold.) In fact, our theorem says that (13) is impossible in square mean.

Furthermore, we can show that the theorem and corollary above are nearly best possible. We will return to this problem also to the proof of (4) in Part II of this paper. 
2. Proof of the Theorem. If an arithmetic function $F(n)$ satisfies (8), then we have

$$
F(N) \geq 1 \text { for } N \geq N_{0} .
$$

Assume first that there exist only finitely many integers $M$ such that

$$
\frac{F(M+j)}{F(M)}<\left(\frac{M+j}{M}\right)^{2} \quad \text { for } j=1,2, \ldots
$$

Then there exists an integer $M_{0}$ such that for $M \geq M_{0}$, there exists an integer $M^{\prime}=M^{\prime}(M)$ satisfying $M^{\prime}>M$ and

$$
\frac{F\left(M^{\prime}\right)}{F(M)} \geq\left(\frac{M^{\prime}}{M}\right)^{2}
$$

Then writing $M_{1}=\max \left(N_{0}, M_{0}\right)$, we get (by induction) that there exist integers $M_{1}<M_{2}<\cdots<M_{j}<\cdots$ such that

$$
\frac{F\left(M_{j+1}\right)}{F\left(M_{j}\right)} \geq\left(\frac{M_{j+1}}{M_{j}}\right)^{2} \quad(\text { for } j=1,2, \ldots)
$$

hence

$$
\begin{gathered}
\frac{F\left(M_{k+1}\right)}{F\left(M_{1}\right)}=\prod_{j=1}^{k} \frac{F\left(M_{j+1}\right)}{F\left(M_{j}\right)} \geq \prod_{j=1}^{k}\left(\frac{M_{j+1}}{M_{j}}\right)^{2}=\left(\frac{M_{k+1}}{M_{1}}\right)^{2}, \\
F\left(M_{k+1}\right) \geq \frac{F\left(M_{1}\right)}{M_{1}^{2}} M_{k+1}^{2} \geq \frac{1}{M_{1}^{2}} M_{k+1}^{2}>M_{k+1}^{3 / 2}
\end{gathered}
$$

for large enough $k$. Obviously,

$$
R(n)=\sum_{a_{1}+a_{j}=n} 1 \leq \sum_{k+l=n} 1=n-1<n
$$

for all $n$. Thus for large $k$ we have

$$
\begin{aligned}
& \Delta\left(M_{k+1}\right)=\sum_{n=1}^{M_{k+1}}(R(n)-F(n))^{2} \geq\left(R\left(M_{k+1}\right)-F\left(M_{k+1}\right)\right)^{2} \\
& =\left(F\left(M_{k+1}\right)\right)^{2}\left(1-\frac{R\left(M_{k+1}\right)}{F\left(M_{k+1}\right)}\right)^{2}>\left(F\left(M_{k+1}\right)\right)^{2}\left(1-\frac{M_{k+1}}{\left(M_{k+1}\right)^{3 / 2}}\right)^{2} \\
& >\frac{1}{2}\left(F\left(M_{k+1}\right)\right)^{2}>\frac{1}{2} M_{k+1}^{3 / 2} F\left(M_{k+1}\right)
\end{aligned}
$$

which proves that (12) cannot hold. 
3. Assume now that there exist infinitely may integers $M$ such that

$$
\frac{F(M+j)}{F(M)}<\left(\frac{M+j}{M}\right)^{2} \quad \text { for } j=1,2, \ldots
$$

We start out from the indirect assumption that $F(n)$ satisfies the assumptions (8), (9) and (10) in the theorem but for a sequence $\mathscr{A}=\left\{a_{1}\right.$ $\left.<a_{2}<\cdots\right\}$ (12) holds. We need the following lemma:

Lemma 1. Assume that $F(n)$ satisfies (8), (9) and (10), (12) holds, $M$ is large enough and it satisfies (15). Put $N=2 M$. Then there exists an integer $d$ such that

$$
0<d \leq 10{ }^{6} F(N)
$$

and

$$
\sum_{\substack{a \leq N \\ a \in \mathscr{A}, a-d \notin \mathscr{A}}} 1>\frac{1}{6}(N F(N))^{1 / 2} .
$$

Proof of Lemma 1. First we give a lower bound for

$$
A(N)=\sum_{\substack{a \leq N \\ a \in \mathscr{A}}} 1
$$

In view of (12), (15) and the assumptions on $F(n)$, and by using Cauchy's inequality, we obtain for large $M$ that

$$
\begin{aligned}
& (A(N))^{2}=\left(\sum_{a_{t} \leq N} a_{l}\right)^{2}=\sum_{a_{t} \leq N, a_{J} \leq N} 1 \geq \sum_{a_{t}+a_{J} \leq N} 1 \\
& \quad=\sum_{n=1}^{N} R(n)=\sum_{n=1}^{N} F(n)+\sum_{n=1}^{N}(R(n)-F(n)) \\
& \quad \geq \sum_{n=M+1}^{N} F(n)-\sum_{n=1}^{N}|R(n)-F(n)| \\
& \geq \sum_{n=M+1}^{N} F(M)-(\Delta(N) N)^{1 / 2}=M F(M)-o\left(N(F(N))^{1 / 2}\right) \\
& >\frac{N}{2}\left(\frac{M}{2 M}\right)^{2} F(2 M)-N(F(N))^{1 / 2} \\
& \quad=\frac{N}{8} F(N)\left(1-(F(N))^{-1 / 2}\right)>\frac{1}{9} N F(N)
\end{aligned}
$$

hence

$$
A(N)>\frac{1}{3}(N F(N))^{1 / 2}
$$


Thus if we denote the number of solutions of

$$
a_{x}-a_{y}=d, \quad a_{x} \leq N
$$

(for fixed $d$ ) by $\varphi(d)$, and $d$ does not satisfy (17), then we have

$$
\begin{aligned}
\varphi(d) & =\sum_{\substack{a \leq N \\
a \in \mathscr{A}, a-d \in \mathscr{A}}} 1=\sum_{\substack{a \leq N \\
a \in \mathscr{A}}} 1-\sum_{\substack{a \leq N \\
a \in \mathscr{A}, a-d \notin \mathscr{A}}} 1 \\
& =A(N)-\sum_{\substack{a \leq N \\
a \in \mathscr{A}, a-d \notin \mathscr{A}}} 1 \geq \frac{1}{6}(N F(N))^{1 / 2} .
\end{aligned}
$$

Starting out from any of the $\left(\begin{array}{c}\Phi(d) \\ 2\end{array}\right)$ pairs $\left(a_{x_{1}}, a_{y_{1}}\right),\left(a_{x_{2}}, a_{y_{2}}\right), x_{1}<x_{2}$ of solutions of (19), we have

$$
a_{x_{1}}-a_{y_{1}}=a_{x_{2}}-a_{y_{2}}, \quad y_{1}<x_{1}<x_{2},
$$

i.e.,

$$
a_{x_{1}}+a_{y_{2}}=a_{x_{2}}+a_{y_{1}}(\leq 2 N), \quad y_{1}<x_{1}<x_{2}
$$

so that in this way we get a pair $\left(a_{u}, a_{v}\right),\left(a_{z}, a_{t}\right)$ satisfying

$$
a_{u}+a_{v}=a_{z}+a_{t}(\leq 2 N), \quad t<u<z .
$$

Furthermore, any pair $\left(a_{u}, a_{v}\right),\left(a_{z}, a_{t}\right)$ satisfying (21) can be obtained twice in this way (from the pairs $\left(a_{u}, a_{t}\right),\left(a_{z}, a_{v}\right)$ and $\left(a_{v}, a_{t}\right),\left(a_{z}, a_{u}\right)$ ). Thus if there does not exist a $d$ satisfying (16) and (17), then in view of (20), the number of solutions of (21) must be at least

$$
\begin{aligned}
\frac{1}{2} \sum_{d=1}^{\left[10^{6} F(N)\right]}\left(\begin{array}{c}
\varphi(d) \\
2
\end{array}\right) & >\frac{1}{2} \sum_{k=1}^{\left[10^{6} F(N)\right]} \frac{\left(\frac{1}{6}(N F(N))^{1 / 2}\right)^{2}}{3} \\
& =\frac{1}{216} N F(N)\left[10^{6} F(N)\right]>1000 N(F(N))^{2}
\end{aligned}
$$

On the other hand, for fixed $n$, the equation

$$
a_{u}+a_{v}=a_{z}+a_{t}=n, \quad t<u<z,
$$

determines one of the $\left({ }_{2}^{R(n)}\right)$ pairs $\left(a_{u}, a_{v}\right),\left(a_{z}, a_{t}\right), u<z$ of solutions of $a_{x}+a_{y}=n$. Thus the number of the pairs $\left(a_{u}, a_{v}\right),\left(a_{z}, a_{t}\right)$ satisfying (21) is at most

$$
\begin{aligned}
& \sum_{n=1}^{2 N}\left(\begin{array}{c}
R(n) \\
2
\end{array}\right) \leq \sum_{n=1}^{2 N} \frac{1}{2}(R(n))^{2} \leq \sum_{n=1}^{2 N}(F(n))^{2}+\sum_{n=1}^{2 N}(R(n)-F(n))^{2} \\
& \quad \leq 2 N(F(2 N))^{2}+\Delta(2 N)=2 N(F(2 N))^{2}+o(N F(2 N)) \\
& \quad<3 N(F(2 N))^{2}=3 N(F(4 M))^{2}<3 N\left(F(M)\left(\frac{4 M}{M}\right)^{2}\right)^{2} \\
& \quad<768 N(F(M))^{2} \leq 768 N(F(N))^{2}
\end{aligned}
$$


This upper bound for the number of pairs satisfying (21) is smaller than the lower bound obtained in (22), and this contradiction proves the existence of an integer $d$ satisfying (16) and (17), which completes the proof of Lemma 1.

4. Throughout the remaining part of the proof, we use the following notations:

$M$ denotes a large integer satisfying (15) and we put $N=2 M . d$ denotes an integer satisfying (16) and (17) in Lemma 1 . We write $e^{2 \pi i \alpha}=e(\alpha)$, and we put $r=e^{-1 / N}, z=r e(\alpha)$ where $\alpha$ is a real variable (so that a function of form $p(z)$ is a function of the real variable $\alpha$ : $p(z)=p(r e(\alpha))=P(\alpha))$. We write

$$
f(z)=\sum_{j=1}^{+\infty} z^{a_{j}}
$$

(By $r<1$, this infinite series and all the other infinite series in the remaining part of the proof are absolutely convergent.) Then we have

$$
f^{2}(z)=\sum_{n=1}^{+\infty} R(n) z^{n}=\sum_{n=1}^{+\infty} F(n) z^{n}+\sum_{n=1}^{+\infty}(R(n)-F(n)) z^{n} ;
$$

hence

$$
\begin{aligned}
\left|f(z)\left(1-z^{d}\right)\right|^{2} \leq & \left|\sum_{n=1}^{+\infty} F(n) z^{n}\right|\left|1-z^{d}\right|^{2} \\
& \quad+\left|\sum_{n=1}^{+\infty}(R(n)-F(n)) z^{n}\right|\left|1-z^{d}\right|^{2}, \\
J= & \int_{0}^{1}\left|f(z)\left(1-z^{d}\right)\right|^{2} d \alpha \\
\leq & \int_{0}^{1}\left|\sum_{n=1}^{+\infty} F(n) z^{n}\right|\left|1-z^{d}\right|^{2} d \alpha \\
& \quad+\int_{0}^{1}\left|\sum_{n=1}^{+\infty}(R(n)-F(n)) z^{n}\right|\left|1-z^{d}\right|^{2} d \alpha \\
= & J_{1}+J_{2} .
\end{aligned}
$$

We will give a lower bound for $J$ and upper bounds for $J_{1}$ and $J_{2}$, and thus also for $J \leq J_{1}+J_{2}$. The lower bound for $J$ will be greater than the upper bound obtained in this way, and this contradiction will prove that the indirect assumption (12) cannot hold, and this will complete the proofs of Theorems 1 and 2. 
5. In this section, we give a lower bound for $J$. We write

$$
f(z)\left(1-z^{d}\right)=\sum_{n=1}^{+\infty} b_{n} z^{n}
$$

Then for $n \in \mathscr{A}, n-d \notin \mathscr{A}$ we have $b_{n}=1$, thus by the Parseval formula and (17) we get

$$
\begin{aligned}
J & =\int_{0}^{1}\left|f(z)\left(1-z^{d}\right)\right|^{2} d \alpha=\sum_{n=1}^{+\infty} b_{n}^{2} r^{2 n} \geq \sum_{n=1}^{N} b_{n}^{2} r^{2 n} \\
& \geq r^{2 N} \sum_{\substack{n \leq N \\
n \in \mathscr{A}, n-d \notin \mathscr{A}}} b_{n}^{2}=e^{-2} \sum_{\substack{n \leq N \\
n \in \mathscr{A}, n-d \notin \mathscr{A}}} 1>\frac{e^{-2}}{6}(N F(N))^{1 / 2} \\
& >\frac{1}{60}(N F(N))^{1 / 2} .
\end{aligned}
$$

6. In this section, we give an upper bound for $J_{1}$. Clearly,

$$
\begin{aligned}
J_{1} & =\int_{0}^{1}\left|\sum_{n=1}^{+\infty} F(n) z^{n}\right|\left|1-z^{d}\right|^{2} d \alpha \\
& =\int_{0}^{1}\left|(1-z) \sum_{n=1}^{+\infty} F(n) z^{n}\right|\left|\frac{1-z^{d}}{1-z}\right|\left|1-z^{d}\right| d \alpha .
\end{aligned}
$$

By (8) and (9), here we have

$$
\begin{aligned}
\mid(1- & z) \sum_{n=1}^{+\infty} F(n) z^{n}|=| F(1) z+\sum_{n=2}^{+\infty}(F(n)-F(n-1)) z^{n} \mid \\
& \leq F(1)+\sum_{n=2}^{+\infty}|F(n)-F(n-1)| r^{n} \\
& \leq c_{3}+\sum_{n=2}^{n_{0}}|F(n)-F(n-1)|+\sum_{n=n_{0}+1}^{+\infty}|F(n)-F(n-1)| r^{n} \\
& =c_{4}+\sum_{n=n_{0}+1}^{+\infty}(F(n)-F(n-1)) r^{n} \\
& =c_{4}+\sum_{n=n_{0}+1}^{+\infty} F(n)\left(r^{n}-r^{n+1}\right)-F\left(n_{0}\right) r^{n_{0}+1} \\
& \left.<c_{4}+(1-r)\left(\sum_{n=n_{0}+1}^{+\infty} F(n) r^{n}\right) \mid \sum_{n=n_{0}+1}^{N} F(n) r^{n}+\sum_{n=N+1}^{+\infty} F(n) r^{n}\right) \\
& =c_{4}+(1-r)
\end{aligned}
$$




$$
\begin{aligned}
< & c_{4}+(1-r)\left(\sum_{n=n_{0}+1}^{N} F(N)+\sum_{n=N+1}^{+\infty} F(N)\left(\frac{n}{N}\right)^{2} r^{n}\right) \\
= & c_{4}+(1-r) N F(N)+N^{-2} F(N) \sum_{n=N+1}^{+\infty} n^{2}\left(r^{n}-r^{n+1}\right) \\
= & c_{4}+(1-r) N F(N) \\
& +N^{-2} F(N)\left(\sum_{n=N+1}^{+\infty}\left(n^{2}-(n-1)^{2}\right) r^{n}+N^{2} r^{N} r^{N+1}\right) \\
< & c_{4}+(1-r) N F(N)+N^{-2} F(N)\left(2 \sum_{n=N+1}^{+\infty} n r^{n}+N^{2}\right) \\
< & c_{4}+(1-r) N F(N)+N^{-2} F(N)\left(2 \sum_{n=1}^{+\infty} n r^{n-1}+N^{2}\right) \\
= & c_{4}+(1-r) N F(N)+2 N^{-2} F(N)(1-r)^{-2}+F(N) \\
= & c_{4}+\left(1-e^{-1 / N}\right) N F(N)+2 N^{-2} F(N)\left(1-e^{-1 / N}\right)^{-2}+F(N) \\
& <c_{4}+N^{-1} N F(N)+2 N^{-2} F(N)(1 / 2 N)^{-2}+F(N) \\
= & c_{4}+10 F(N)<11 F(N) .
\end{aligned}
$$

Furthermore, we have

$$
\begin{aligned}
&\left|\frac{1-z^{d}}{1-z}\right|=\left|\sum_{j=0}^{d-1} z^{j}\right| \leq \sum_{j=0}^{d-1}\left|z^{J}\right|<\sum_{j=0}^{d-1} 1=d \text { for all } \alpha \\
&|1-z|=((1-z)(1-\bar{z}))^{1 / 2}=\left(1+|z|^{2}-2 \operatorname{Re} z\right)^{1 / 2} \\
&=\left(1+r^{2}-2 r \cos 2 \pi \alpha\right)^{1 / 2}=\left((1-r)^{2}+2 r(1-\cos 2 \pi \alpha)\right)^{1 / 2} \\
&>(2 r(1-\cos 2 \pi \alpha))^{1 / 2}=\left(2 e^{-1 / N} \cdot 2 \sin ^{2} \pi \alpha\right)^{1 / 2} \\
& \geq\left(2 \cdot \frac{1}{2} \cdot 2 \cdot(2 \alpha)^{2}\right)^{1 / 2}=\left(8 \alpha^{2}\right)^{1 / 2} \geq 2 \alpha \text { for } 0 \leq \alpha \leq \frac{1}{2}
\end{aligned}
$$

so that

$$
\left|\frac{1-z^{d}}{1-z}\right| \leq \frac{1+\left|z^{d}\right|}{|1-z|}<\frac{2}{2 \alpha}=\frac{1}{\alpha} \quad \text { for } 0<\alpha \leq \frac{1}{2}
$$

and

$$
\left|1-z^{d}\right| \leq 1+\left|z^{d}\right|<2 \text { for all } \alpha \text {. }
$$


By (8), (10), (16), (18), (26), (27) and (28), we obtain from (25) that for large $N$,

$$
\begin{aligned}
J_{1} & \leq \int_{0}^{1} 11 F(N)\left|\frac{1-z^{d}}{1-z}\right| \cdot 2 d \alpha=44 F(N) \int_{0}^{1 / 2}\left|\frac{1-z^{d}}{1-z}\right| d \alpha \\
& =44 F(N)\left(\int_{0}^{1 / d}\left|\frac{1-z^{d}}{1-z}\right| d \alpha+\int_{1 / d}^{1 / 2}\left|\frac{1-z^{d}}{1-z}\right| d \alpha\right) \\
& <44 F(N)(1+\log d) \leq 44 F(N)\left(1+\log 10^{6} F(N)\right) \\
& <45 F(N) \log F(N)<45 F(N) \log 9(A(N))^{2} N^{-1} \\
& \leq 45 F(N) \log 9 N^{2} \cdot N^{-1}<46 F(N) \log N \\
& =46(N F(N))^{1 / 2}\left(F(N) N^{-1}(\log N)^{2}\right)^{1 / 2}=46(N F(N))^{1 / 2} o(1) \\
& =o(N F(N))^{1 / 2} .
\end{aligned}
$$

7. In this section, we estimate $J_{2}$ and we complete the proof of the theorem.

By using (8), (9), (15), the indirect assumption (12), Cauchy's inequality and the Parseval formula, we obtain that if $N$ is large enough in terms of $\varepsilon$ then

$$
\begin{aligned}
& J_{2}=\int_{0}^{1}\left|\sum_{n=1}^{+\infty}(R(n)-F(n)) z^{n}\right|\left|1-z^{d}\right|^{2} d \alpha \\
& \leq \int_{0}^{1}\left|\sum_{n=1}^{+\infty}(R(n)-F(n)) z^{n}\right|\left(1+|z|^{d}\right)^{2} d \alpha \\
& \leq 4\left(\int_{0}^{1}\left|\sum_{n=1}^{+\infty}(R(n)-F(n)) z^{n}\right|^{2} d \alpha\right)^{1 / 2} \\
& =4\left(\sum_{n=1}^{+\infty}(R(n)-F(n))^{2} r^{2 n}\right)^{1 / 2} \\
& =4\left(\sum_{n=1}^{+\infty}(\Delta(n)-\Delta(n-1)) r^{2 n}\right)^{1 / 2}=4\left(\sum_{n=1}^{+\infty} \Delta(n)\left(r^{2 n}-r^{2 n+2}\right)\right)^{1 / 2}
\end{aligned}
$$




$$
\begin{aligned}
& =4\left(\left(1-r^{2}\right) \sum_{n=1}^{+\infty} \Delta(n) r^{2 n}\right)^{1 / 2} \\
& <4\left(\left(1-r^{2}\right)\left(c_{5}+\sum_{n=1}^{+\infty} \varepsilon^{2} n F(n) r^{2 n}\right)\right)^{1 / 2} \\
& <4\left(\left(1-r^{2}\right)\left(c_{5}+\varepsilon^{2} \sum_{n=1}^{N} N F(N) r^{2 n}+\varepsilon^{2} \sum_{n=N+1}^{+\infty} n F(n) r^{2 n}\right)\right)^{1 / 2} \\
& <4\left(\left(1-e^{-2 / N}\right)\left(c_{5}+\varepsilon^{2} N^{2} F(N)\right)\right. \\
& \left.+\varepsilon^{2}\left(1-r^{2}\right) \sum_{n=N+1}^{+\infty} n F(M+(n-M)) r^{2 n}\right)^{1 / 2} \\
& <4\left(2 \cdot \frac{2}{N} \varepsilon^{2} N^{2} F(N)+\varepsilon^{2}\left(1-r^{2}\right) \sum_{n=N+1}^{+\infty} n F(M)\left(\frac{n}{M}\right)^{2} r^{2 n}\right)^{1 / 2} \\
& <4\left(4 \varepsilon^{2} N F(N)+4 \varepsilon^{2} F(N) N^{-2} \sum_{n=N+1}^{+\infty} n^{3}\left(r^{2 n}-r^{2 n+2}\right)\right)^{1 / 2}
\end{aligned}
$$

(where $c_{5}=c_{5}(\varepsilon)$ ). Here we have

$$
\begin{aligned}
& \sum_{n=N+1}^{+\infty} n^{3}\left(r^{2 n}-r^{2 n+2}\right)=N^{3} r^{2(N+1)}+\sum_{n=N+1}^{+\infty}\left(n^{3}-(n-1)^{3}\right) r^{2 n} \\
& \quad<N^{3}+\sum_{n=N+1}^{+\infty} 4 n^{2} r^{2 n}=N^{3}+4\left(1-r^{2}\right)^{-1} \sum_{n=N+1}^{+\infty} n^{2}\left(r^{2 n}-r^{2 n+2}\right) \\
& =N^{3}+4\left(1-e^{-2 / N}\right)^{-1}\left(N^{2} r^{2(N+1)}+\sum_{n=N+1}^{+\infty}\left(n^{2}-(n-1)^{2}\right) r^{2 n}\right) \\
& <N^{3}+4 N\left(N^{2}+2 \sum_{n=N+1}^{+\infty} n r^{2 n}\right) \\
& =5 N^{3}+8 N\left(1-r^{2}\right)^{-1} \sum_{n=N+1}^{+\infty} n\left(r^{2 n}-r^{2 n+2}\right) \\
& =5 N^{3}+8 N^{2 n}\left(1-e^{-2 / N}\right)^{-1}\left(N r^{2(N+1)}+\sum_{n=N+1}^{+\infty} r^{2 n}\right) \\
& <5 N^{3}+8 N^{2}\left(N+4^{2(N+1)}\left(1-r^{2}\right)^{-1}\right) \\
& <13 N^{3}+8 N^{2}\left(1-e^{-2 / N}\right)^{-1} \\
& <13 N^{3}+8 N^{3}=21 N^{3} .
\end{aligned}
$$


Thus we obtain from (30) that for $N$ large enough

$$
\begin{aligned}
J_{2} & <4\left(4 \varepsilon^{2} N F(N)+4 \varepsilon^{2} F(N) N^{-2} \cdot 21 N^{3}\right)^{1 / 2} \\
& =4\left(88 \varepsilon^{2} N F(N)\right)^{1 / 2}<40 \varepsilon(N F(N))^{1 / 2} .
\end{aligned}
$$

By (23), (24), (29) and (31), we get that if $N$ is large enough (in terms of $\varepsilon$ ) then

$$
\begin{aligned}
\frac{1}{60}(N F(N))^{1 / 2} & <J \leq J_{1}+J_{2}<\varepsilon(N F(N))^{1 / 2}+40 \varepsilon(N F(N))^{1 / 2} \\
& =41 \varepsilon(N F(N))^{1 / 2}
\end{aligned}
$$

But for $\varepsilon=1 / 2500$ (and if $N$ is large so that $F(N)>0$ by (14)) this inequality cannot hold. This contradiction proves that (12) cannot hold which completes the proof of the theorem.

\section{REFERENCES}

[1] P. Erdős, Problems and results in additive number theory, Colloque sur la Théorie des Nombres (CBRM) (Bruxelles, 1956), 127-137.

[2] P. Erdős and A. Rényi, Additive properties of random sequences of positive integers, Acta Arithmetica, 6 (1960), 83-110.

[3] H. Halaberstam and K. F. Roth, Sequences, Springer-Verlag, 1983.

Received September 12, 1984.

Mathematical Institute

Hungarian ACADEMy OF SCIENCES

1053 BUdAPEST, REÁltaNODa U. 13-15

HUNGARY 
